\title{
Searching for Mental Health: A Mixed Methods Study of Young People's Online Help-seeking
}

\author{
Claudette Pretorius ${ }^{1}$ Darragh McCashin $^{2}$ Naoise Kavanagh $^{3}$ David Coyle $^{1}$ \\ ${ }^{1}$ School of Computer Science, \\ University College Dublin, \\ Ireland \\ ${ }^{2}$ School of Psychology, \\ University College Dublin, \\ Ireland \\ ${ }^{3}$ Jigsaw, National Centre for \\ Youth Mental Health, \\ Dublin, Ireland
} claudette.pretorius@ucdconnect.ie, \{darragh.mccashin,d.coyle\}@ucd.ie,naoise.kavanagh@jigsaw.ie

\begin{abstract}
Seeking help is often an important step in addressing mental health difficulties. Evidence suggests that positive helpseeking experiences contribute to an increased likelihood of future help-seeking and achieving improved outcomes. However, help-seeking is a complex process. Alongside traditional sources, digital technologies offer many pathways to help. Using a mixed methods approach across two studies, this paper explores key design factors for online mental health resources that can support young people's helpseeking. First, a large online survey $(n=1308)$ highlighted challenges and identified common help-seeking scenarios, including information-seeking, person-centred approaches and crisis situations. Using survey data, personas were developed to represent different help-seekers - each characterised by a particular help-seeking scenario. The personas were then used in co-design workshops to facilitate further exploration of help-seeking needs. Four key design considerations were identified: connectedness, accessible information, personalisation, and immediacy. Based on our findings, we provide design recommendations that are grounded in existing theories of help-seeking.
\end{abstract}

\section{Author Keywords}

Mental health; help-seeking; search; young people;

behaviour; co-design; personas; mixed methods

\section{CSS Concepts}

-Human-centered computing Human computer interaction (HCI);

\section{INTRODUCTION}

The prevalence of mental health difficulties in the general population is well documented $[12,58]$, with the age of onset for most psychiatric disorders falling within the $14-25$ age group [33]. Young people face a number of challenges that are unique to their developmental stage, including concerns about body image, education, and transitioning into

Permission to make digital or hard copies of all or part of this work for personal or classroom use is granted without fee provided that copies are not made or distributed for profit or commercial advantage and that copies bear this notice and the full citation on the first page. Copyrights for components of this work owned by others than ACM must be honored. Abstracting with credit is permitted. To copy otherwise, or republish, to post on servers or to redistribute to lists, requires prior specific permission and/or a fee. Request permissions from Permissions@acm.org.

CHI 2020, April 25-30, 2020, Honolulu, HI, USA.

(C) 2020 Copyright is held by the owner/author(s). Publication rights licensed to

ACM. ACM ISBN 978-1-4503-6708-0/20/04 ..\$15.00.

DOI: https://doi.org/10.1145/3313831.337632 independent living [29, 38]. As with many health-related concerns, there are barriers to accessing the appropriate help when necessary, including stigma around accessing mental health care, long waiting periods to see a mental health professional, financial constraints, and concerns around privacy and confidentiality $[13,14,51]$. Technology can and has played a role in addressing some of the traditional barriers young people can face when seeking help for mental health issues.

For many young people, particularly in developed countries, use of the Internet is embedded in their day-to-day lives. Evidence suggests young people are increasingly using the Internet to access mental health information [4, 20, 57]. They can do so anonymously, are not limited by geographical location, and a wide variety of information is readily available [9]. These factors can contribute to young people having increased access to resources, as well as increased control in meeting their own mental health needs [31]. Unfortunately, the quality of online mental health resources, both formal and informal, is inconsistent [37]. These resources can impact the psychological wellbeing of a young person and influence their likelihood of future help-seeking. It has been found that future help-seeking intentions are improved if previous help-seeking experiences have been positive [22]. Although some data exist about young people's online help-seeking preferences, little is known about young people's patterns of help-seeking online and how their needs can be appropriately met.

In this paper, we explore young people's online help-seeking practices. We begin by providing empirical evidence on how young people who are experiencing mental health concerns seek help online. Building on this, we worked directly with young people to explore how web-based technology can better meet their help-seeking needs. Two separate studies are presented. Study 1, a large online survey, explored young people's help-seeking preferences, patterns of Internet use for mental health, sources of information, in addition to satisfaction with content accessed online. The study identified three distinct scenarios or categories of helpseeker, which we subsequently translated into personas. In Study 2, these personas were used in co-design workshops with young people to gain further insight into online helpseeking journeys. Methodologically, personas proved 
valuable - allowing for engagement with sensitive topics in a group setting, without the need for direct personal disclosure.

This paper makes several distinct contributions. It provides empirical evidence of the approaches taken and challenges faced when young people use the Internet to seek help for mental health difficulties. Three key help-seeking scenarios are identified: self-directed information-seeking; personcentred approaches that emphasis relatedness and personal connections; and crisis situations. Building on this, personas provided a concrete means to investigate these help seeking scenarios, via co-design with young people. Four important considerations are identified that can help to support a positive help-seeking experience: connectedness; accessible information; personalization; and immediacy (i.e. options for immediate action). Based on our findings, we provide theoretically grounded recommendations to support the design of online help-seeking resources.

\section{RELATED WORK}

Within the mental health field, help-seeking has been defined as "an adaptive coping process that is the attempt to obtain external assistance to deal with a mental health concern" [52]. Help-seeking sources can be classified as either informal or formal [59]. In an offline context, informal sources are those sources such as peers, partners, friends and family. Formal sources are those sources with whom the person does not share a personal relationship and are usually from a professional, such as a general practitioner, psychologist or educator [59]. Historically, young people have shown a preference for seeking help from informal sources and a greater preference for self-reliance [55]. These same classifications can be applied to online resources, where health websites, online counselling services and charity websites would be considered to be formal online sources. Formal sources can also be accessed across various platforms including social media channels such as Instagram and Twitter. Similarly, informal sources can be accessed on these same platforms; as well as through unmoderated discussion forums, blogs and vlogs hosted by peers, and unclassified websites [50]. Traditionally, barriers to offline help-seeking would be fear of stigma, difficulty in accessing services and preferences for self-reliance [22]. Online helpseeking provides the opportunity to overcome these barriers $[21,31]$. Previous research has found that help-seeking can be facilitated by having positive help-seeking experiences; having social support and encouragement from others, and improved mental health literacy [55]. Through thoughtful and critical design, online resources can support these facilitators and address the traditional barriers to help-seeking for mental health concerns.

In this paper we explore online help-seeking via the lens of existing offline help-seeking theory. We recognise that this issue could also be approached via an Information Science perspective, building on work by researchers such as Fisher et al. [17, 18] and Rieh [28, 53], but this was beyond the scope of the current paper. The potential to combine these different theoretical perspectives is discussed further in our Limitations and Future Work section.

\section{Online Help-seeking Practices}

Previous research has focused on the content of social media posts and Google search-logs to predict mental health conditions or to be used as tools to assist with health assessment $[11,60]$. More recently, research has begun to focus on how the Internet and social media is being used to find support for mental health difficulties. A study by Andalibi [3] found that Instagram was being used by people experiencing depressive symptoms to connect with others undergoing similar experiences and to seek support. Many young people are also making use of search engines and specific mental health resource websites to meet their needs. Individual search-logs can be particularly insightful of the difficulties currently being experienced. A study by Zaman et al [60] shows that individual search-logs can provide indicators that can identify those struggling with low selfesteem. Additionally, Birnbaum et al [5] found that the search terms used by young people vary depending on the mental health difficulty faced. Those with psychotic disorders were more likely to search why their symptoms had started, whereas those with mood disorders were more likely to search for how to stop their symptoms. Young people most at risk, those experiencing self-harm or suicidal intent, are more likely to access the Internet than reach out to someone offline [19]. A slightly larger proportion of these young people are accessing resources that are helpful but there is still a proportion accessing potentially harmful sites [34]. A systematic review by Kauer et al [31] investigating the effectiveness of online interventions on help-seeking behaviour found that, despite most studies reporting that young people were satisfied with online mental health resources, very few studies reported the effect of these online resources on facilitating young people's help-seeking.

\section{Theoretical Models of Help-seeking}

Several existing theories provide conceptual frameworks to understand help-seeking. While each theory was developed in the context of offline help-seeking, they can provide valuable building blocks to also understand online helpseeking. The studies described below apply Rickwood's theory and Self Determination Theory (SDT). Rickwood's work has been widely applied to young people in offline contexts. It provides a stage-based approach to analysis. In contrast SDT focuses on basic psychological needs. Together they provided a means to investigate basic needs at different stages of an online help-seeking process. Rickwood's model and questionnaires are applied in the design of Study 1. Both theories are applied in the analysis of data in Studies 1 and 2. For completeness we also include a brief discussion of the Theory of Planned behaviour, although this specific theory was not applied in our work.

\section{Rickwood's Help-seeking model}

Rickwood's model is a stage-based model that reflects the interpersonal processes that take place when a young person seeks help [51]. It recognises the internal process that needs 
to take place for help-seeking to move from an intention to a behaviour; from the intrapersonal to the interpersonal. These stages are: awareness and appraisal of problems; expression of symptoms and need for support; availability of sources of help; and willingness to seek out and disclose [59]. This model was specifically developed for help-seeking for mental health difficulties in a population age range of $14-24$ years in the offline context. This model takes into account that help-seeking can be formal or informal but fails to take into account some of the external barriers that can hinder helpseeking [30].

\section{Theory of Planned Behaviour}

Although not applied in this paper, this model proposes that behaviour is determined by behavioural intentions [1]. A person's behavioural intentions are informed by: their attitude toward the behaviour; their subjective norms around the execution of the behaviour; and their perceived behavioural control over the behaviour [1]. It has been suggested that, by changing a person's attitude towards helpseeking through improving their mental health literacy and changing their subjective norms (i.e. reducing the stigma), you can positively influence their intention to seek help [7]. However, the change from intention to behaviour is moderated by the individual's perceived behavioural control, which in the case of help-seeking are factors such as the availability of appropriate services; cost of services and accessibility of services [28]. This model takes into account the barriers that may hinder help-seeking but is not specific to a particular behaviour.

\section{Self-Determination Theory}

Self-Determination Theory (SDT) is a theory of motivation and wellbeing [54]. Central to this theory is the concept of three basic psychological needs: competence, relatedness and autonomy; that when satisfied will promote wellbeing and sustained motivation [54]. It also asserts that goal pursuits can be understood through the satisfaction of these three psychological needs and that facilitators and barriers to a behaviour can be understood in terms of fulfilment of these needs [16]. SDT is being successfully applied to the development of technologies and is useful to apply when trying to understand user's design needs $[10,48]$. A study by Naqshandi et al [39] investigating online peer supporter's design needs for a chat system with early mental health helpseekers, found that the themes from the data aligned with the three basic psychological needs outlined above. SDT can be used to understand motivation in online help-seeking, assuming that where all three needs are met, it could move an individual towards their intended help-seeking goal and enhance their wellbeing.

\section{A MIXED METHODS APPROACH}

This research applies a mixed methods approach, including quantitative and qualitative data, and combining an initial online survey (Study 1) with later co-design workshops (Study 2). In designing and recruiting for our studies, we recognised that young people seeking help are often doing so in relation to everyday stressors. As a result, we deliberately sought to de-medicalise the language we used. For example, the online survey addressed emotional wellbeing instead of using the term mental health. This decision was based on early feedback from youth panels that helped to inform the design of our studies. Similarly, the co-design workshops made extensive use of personas, rather than asking young people to speak directly to personal experience.

It is common for HCI research in healthcare to use co-design with specific diagnostic populations in order to ensure that user needs are met $[24,56]$. However, this research sought to gain the perspective of young people who fall into the general population and might not fall into any clinical categories, as would be the case for many young people searching for help online. Co-design methods investigating design needs with young people in relation to mental health have been found to be very useful in the past [32, 40]. They provide a medium to build a shared understanding based on research findings; but can also help to gain the perspective of potential users in a non-intimidating manner [23]. Previous HCI research has demonstrated that the use of personas is an acceptable way to facilitate empathy and help to focus design sessions [35, 36, 43].

\section{STUDY 1: ONLINE SURVEY}

\section{Procedure}

This was an exploratory study which investigated the online help-seeking behaviours and preferences of young people aged 18-25, living in Ireland. We made use of an anonymous online survey hosted on LimeSurvey. Participants were recruited through social media ads on Twitter and Facebook. The survey consisted of both open and closed questions and addressed young people's technology use, their propensity to seek help from different sources, current personal and emotional concerns, preferred online resources, the credibility of online resources, and positive and negative experiences of online help-seeking. Quantitative data were analysed using descriptive statistics in IBM SPSS Statistics for Mac, Version 24; and qualitative data were analysed using QSR International's NVivo 12 software and was inductively coded using thematic analysis following Braun \& Clarke's methodology [8]. All of the data was coded by the first author, with the second and fourth author coding a percentage of the data. Disagreements were discussed until consensus was reached.

The full quantitative results of this study are reported in [49]. Here we provide a brief summary to provide context for our qualitative work and subsequent discussion of our co-design study (Study 2). The qualitative results of this study have not been reported previously. Ethics permission for this study was provided by University College Dublin Office of Research Ethics (LS-17-116-Pretorius-Coyle).

\section{Results}

Survey participants

A total of 1308 participants completed the online survey, of which $78.52 \%(\mathrm{n}=1027 / 1308)$ were female, $18.50 \%(\mathrm{n}=$ 
242)/1308 were male, $1.68 \%(\mathrm{n}=22 / 1308)$ were non-binary and $0.84 \%(\mathrm{n}=11 / 1308)$ identified as transgender. The mean age of the population was $20.68(s d=2.22)$, with a minimum and maximum age of 18 and 25 respectively. The survey had good national coverage. Of the whole sample, $67.13 \%$ $(n=878 / 1308)$ reported that they were currently living in a city or town. Most of the sample reported their current level of education to be undergraduate $(59.17 \%, \mathrm{n}=774 / 1308)$. A majority of the sample accessed the survey link through Twitter $(68.88 \%, \mathrm{n}=901 / 1308)$ and Facebook $(24.08 \%$, $\mathrm{n}=315 / 1308$ ).

\section{Quantitative Results}

The survey showed that school/college (87\%), body image $(73 \%)$ and exams $(72 \%)$ were personal concerns for the majority of the sample. The results further indicated that there was a correlation between these personal concerns and young people looking for help online. The survey demonstrated that the majority $(82.6 \%)$ of the sample would make use of an Internet search to look for help online, however only $36.9 \%$ of the respondents were satisfied with their experience of using search. Other resources used by young people included health websites or discussion forums. Levels of satisfaction were higher for health websites, with $49.3 \%$ of the sample indicating that they were satisfied with this experience when help-seeking. Very few young people $(12.2 \%)$ would make use of a mental health app. Open response questions in this survey indicated that other sources accessed when help-seeking could be classified in the following ways: formal offline source, informal offline source, formal online source and informal online source. When surveyed on the credibility of online resources, health websites were deemed trustworthy by $39.5 \%$ of the sample; with $47.1 \%$ indicating that they found an Internet search untrustworthy. Overall, none of the online sources listed were rated as 'trustworthy' or 'very trustworthy' by the majority of respondents. Respondents provided insight into which elements they deemed as important indicators of credibility online. This included: a health service logo; endorsement by a school or college; the presence of another governmental logo; and reference to scientific data or authors. In an open response question, young people indicated the following as playing a role in promoting credibility: links to local support services; grounded in research; design and layout; quality of content; the ability to rank or comment on content; and the ability to contact someone directly through the online resource.

When asked about facilitators and barriers to help-seeking, young people confirmed that anonymity and confidentiality played an important role in their motivation to use the Internet for help-seeking. Additionally, being able to connect with others with similar experiences was a significant facilitator. Some of the barriers included not knowing if information found online was reliable $(55.9 \%)$ and a preference for self-reliance $(52.7 \%)$. Respondents also had worries about their privacy and anonymity (32.1\%) and concerns that others might find out (43\%).

\section{Qualitative Results}

The qualitative sections of the survey focused on young people's experiences, in particular the barriers and facilitators they experience in seeking help online and their attitudes towards information-based help versus connection with another person. For example, participants were asked "Is being able to connect to another person important to you when seeking help online? If yes, why?" 536 participants answered this question.

Qualitative analysis identified affordability, anonymity, ease of access, validation of experience, reduced stigma, and privacy as key facilitators to online help-seeking. For example, a participant wrote:

I often go online because I know there is something wrong, but I don't want to tell anyone in my real life for fear that they will judge me or they won't care and i'll just be bothering them. Online help can help me deal with my problem alone so I will not have to tell anyone.

Affordability, lack or personalization, lack of mental health literacy and concerns about the credibility of online resources were key barriers to a positive experience. Participants comments included:

It's not personal: all the information out there already exists and is not tailored for me.

Being unsure what to search/look for online... searching for hours for a website that I am comfortable with.

Analysis of questions around help-seeking preferences revealed a number of key groupings. The information-seeker values independence, their objective is to browse information regarding their mental health query without the intervention or interference of another person, online or offline. This type of help-seeker wants a confidential experience that allows them to access well-written, credible information. This is evidenced through comments such as "I prefer to be independent" and "I like to gather the information myself, without talking to others".

In contrast, other help-seekers are person-centred. They are looking for an affirming and empathetic experience. This help-seeker wants to validate their own experiences and would like to make use of an online guide. This could be due to feelings of uncertainty, "Sometimes it can be hard to identify what the problem is," as well as wanting a personalised experience:

I think a lot of information is very general and can seem cold and clinical. If you can talk to someone about your personal and specific experience, information can be tailored and make the person feel more important.

Finally, people seek help online when they are facing $\boldsymbol{a}$ crisis situation. This help-seeker wants to connect with another person, either online of offline, because they are in severe distress and need assistance: 
Sometimes, in very severe situations I want to talk to a real person...

Often a crisis help-seeker is looking for immediate relief ("it is immediate"). But when they are looking for help and a personal connection, it is often outside of typical office hours ("I find this especially true at night time, and this is the time when all of the usual go-to help are not available").

Our data suggests that many online help-seekers will fall into one of three groupings identified above. However, it is also clear that people cannot, and should not, be referenced as a single static type of help seeker. Rather, young people seek different types of help at different times in their lives, depending on their needs and goals. For example:

It can be useful during times of crisis and extreme emotional stress, but with average emotional stress I prefer to do my own research.

Finally, many young people indicated that when they seek help online, it is often in regard to the needs of another person, e.g. a friend or family member. The needs of the third-party help-seeker therefore also need to be considered.

\section{Discussion}

The findings from the survey suggest four broad categories of design features that can either meet or frustrate an online help-seeker's needs.

\section{Content}

The spectrum of help-seeking needs is broad and thus the types of content provided by an online resource should cater for this. The survey found that young people do make use of information pages containing factual information about symptoms and treatment. Less formal information was also favoured and can be provided through the use of personal stories detailing the lived experiences of other young people. To address the objectives of different types of help-seekers, young people indicated that reliable information regarding self-help strategies should be included in online resources. These strategies need to be supported by research, and references to research should be included. Content should be tailored so that it is applicable to both people searching for themselves and those searching for others.

\section{Interactive Features}

The survey highlighted that reading is not the only activity engaged in by online help-seekers. There is a need to enable them to interact and engage with the content they find online. Users suggested that this could be done through comments sections at the bottom of information pages with the ability to upvote certain comments, similar to platforms such as Reddit. Depending on the needs of the help-seeker, the need to connect with another person online whilst help-seeking was highlighted. This can be with a peer or a professional. Finally, any resource needs to be designed for mobile. Good design needs to create a comfortable environment for online content and provide features that are easily accessed through mobile technology.

\section{Support Credibility}

Concerns over credibility are likely to become a barrier to further help-seeking in the future. Helping young people to assign credibility to online resources also has the potential to play an important role in developing their mental health literacy. The findings from the survey indicate that young people value information that is backed by research and written by experts in the field. Young people's online information-gathering behaviours can be facilitated by including hyperlinks to other online resources that can complement the help-seeking journey. Online resources can indicate their credibility by working in partnership with governmental health services and local schools or colleges.

Support Different Help-Seeking Pathways and Different Kinds of Help-Seekers

Online help-seeking pathways for different help-seekers need to be considered. Existing online resources are predominantly guided by mental health difficulty, diagnoses and symptoms. This has the risk of pathologizing normal emotional responses to everyday life events and stressors which may negatively impact help-seekers experience, deterring future help-seeking when in need.

\section{PERSONA DEVELOPMENT}

Study 1 allowed us to collect data from a large number of young people but relied on online survey methods. In Study 2 , we applied co-design methods to engage more directly with young people. As mental health is a sensitive subject and co-design involved group workshops, we felt it would be inappropriate for the participants to speak directly to their own mental health experiences. Instead, data from Study 1 was used to develop empirically informed personas for young people seeking help for mental health difficulties. Each persona was characterised by one of three goals: to find information; to connect with others, and to find immediate help in times of crisis.

Persona development was informed by Nielsen's description of engaging personas [41]. Engaging personas make use of stories to provide an understanding of the whole person and avoid seeing the user as a stereotype [41, 43]. Engaging personas also take into consideration the emotions and backgrounds of the user. This perspective emphasizes the narratives of the user and how stories can engage and bring personas to life. Nielsen [41, 42, 43, 44] outlines a ten-step process to create these personas which can be summarised by four main sections: data collection and analysis; persona descriptions; scenarios for problem analysis and idea development; and finally, acceptance from the organisation and involvement of the design teams. In the first three steps, quantitative data (in this case, the survey data) was used to determine who the users are and what they do with the current systems. A hypothesis was then formed to describe different user groups. These hypotheses are then verified. For this study, qualitative data from the survey study and previous systematic review of the literature was used to accomplish this [50]. As part of step 4, it was decided to create one persona per grouping identified in Study 1. Once 


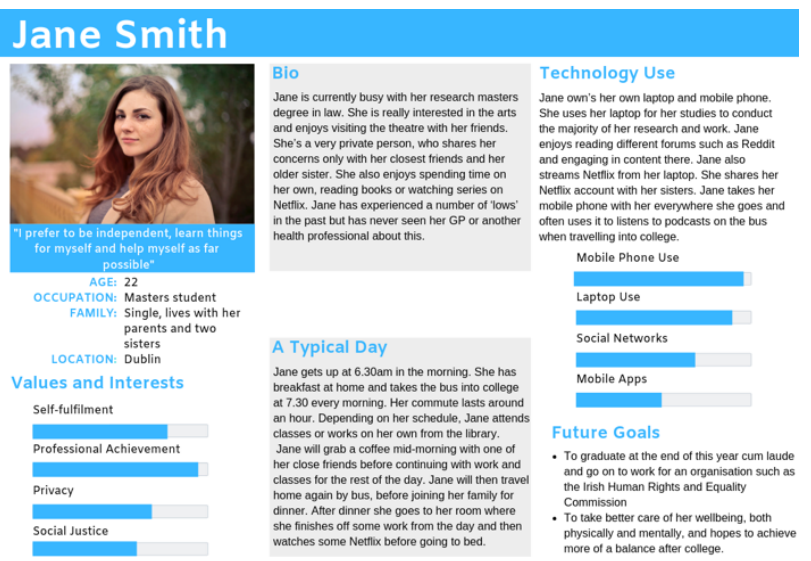

Figure 1. Jane represents the Information Seeker.

the number of personas were decided, steps 5 and 6 included describing these personas and preparing scenarios for each persona. These were again informed by the results of the survey. Throughout the process, designs were discussed among team members and field experts, to ensure they were valid and representative of the data and actual users.

Figure 1 shows one of the final personas. All three personas are included as supplementary materials. Beyond the scope of this paper, the personas may be useful to other researchers working in this field. They are empirically derived and can help to inform both design processes and decisions.

\section{STUDY 2: CO-DESIGN WORKSHOPS}

\section{Procedure}

Four separate, two-hour co-design workshops took place over a one-month period with four different groups in different urban and rural locations. Two of the workshops took place with students from local vocational colleges, with the final two workshops taking place with young people from a charity youth advisory panel. All recruitment was facilitated by a youth mental health charity. There were a total of 35 (Female $=20$, Male $=15)$ participants across all workshops. Co-design consisted of three distinct activity phases and the format was informed by the work of Blake et al [6]. Data was collected in the form of audio recordings of group interactions and artefacts created by groups during the workshops. Artefacts were later photographed, and audio recordings transcribed. Co-design study participants were not asked about their personal experience of mental health services, due to concerns that confidentiality might be compromised in a group setting. Ethics permission for this study was provided by University College Dublin Office of Research Ethics (LS-19-20-Pretorius-Coyle).

\section{Activity One: Persona Immersion}

The three personas were used to provide representative illustrations of online help-seekers, according to the groupings described earlier. Participants were divided into groups of two or three, with each group assigned one persona. The intent was to allow participants to become immersed with the user and allow them to think of the help- seeking journey from their perspective. To facilitate this, groups were asked to work through the scenario from the point of view of the persona. Participants were asked to complete an empathy map to elicit empathy and understanding [15]. The personas and empathy maps allowed participants to explore a sensitive topic from the perspective of another young person.

\section{Activity Two: Mapping the help-seeking journey}

In the same small groups, participants were then asked to map the help-seeking journey for each persona using the scenarios [26]. The groups were asked to execute the steps the persona might take to achieve their personal help-seeking objectives and to record these steps. Participants were asked how the person would feel at different points in the process; where they may feel their needs are met and where that particular persona might feel frustrated.

\section{Activity Three: Brainstorming features for an online mental} health resource

The brainstorming section of the workshop consisted of two parts. The participants were first asked to brainstorm as many features and qualities they thought were relevant for an online mental health resource for their persona. They completed this brainstorming activity on their own and then discussed their ideas in their groups and grouped similar ideas together. Once this was completed, participants were then asked to sketch their most preferred features. To conclude, participants discussed their sketches in groups and identified the most important features overall.

\section{Activity Four: Designing the online experience}

Each small group was asked to design the ideal online mental health resource that would facilitate the help-seeking of their persona and which incorporated the features they had identified in the brainstorming process. Participants used A1sized paper and markers to illustrate their designs.

\section{Data Analysis}

A directed content analysis approach was used on the qualitative data [27]. All workshop artefacts were photographed and uploaded to NVivo. Three of the authors participated in coding. The first author coded all workshop data using open and closed coding. An a priori list of codes were derived from the Rickwood's help-seeking model, SelfDetermination Theory, known facilitators and barriers to help-seeking, and categories of features that would inform design. Data that could not be coded to the predetermined codes were identified and analysed to determine whether they represented a new category or sub-category. The two remaining authors used the same approach, with the second author coding $75 \%$ of the data and the last author coding $25 \%$ of the data. Thereafter, codes were compared, and any disagreements were discussed until consensus was reached. Using this qualitative approach, each artefact could be coded for multiple codes. Once coding was completed, codes from facilitator and barrier categories, as well as from feature categories, were analysed for overall themes. 


\section{Results}

Collectively, the overall dataset clustered into four key themes that described important design needs for young people throughout their help-seeking journey.

\section{Chat and relatedness}

Participants repeatedly described chat features as an important component to help-seeking and expressed a clear wish for relatedness. This was described in a variety of formats, including virtual reality, chatbot-driven, 24/7 realtime interactions, community forums, options for chat with both peers and professionals, in addition to preferences for anonymous chat. Across all workshop designs, participants included a chat option within a broader interface that addressed various help-seeking pathways. The chat function was also highlighted by some of the participants as a useful way to assist those who were in crisis.

The only thing about a live chat, if it's $24 / 7$, it would have to be like, you can feel bad at any time in the day like, you know what I mean? Maybe like you could have an emergency contact thing, where it's like, if you go on at two in the morning, like someone, like you could go on a call or something.

Links to social media were also a minor theme within the data. Participants used social media as a means to connect to a broader community as well as means to learn about online mental health resources.

\section{Information and competence}

The second key theme was a desire for information regarding mental health issues, noting also the impact that too much information could have on a person's sense of competence. Participants showed strong preferences for traditional information page content including articles and FAQs. Many groups named formal websites (such as Healthline or the National Health Service in the UK). They were also clear in expressing their wish for diverse formats of information videos, podcasts, links to other sources, and personal stories were all seen as helpful.

Connected to the drive for information was the need for accessible layouts and design, and this was seen as integral to both the credibility and usability of the online resources. Furthermore, participants indicated that credibility could be promoted by referencing scientific research and mental health professionals.

The issue of competence was most clear when participants discussed strategies of searching for information. Activity two asked all groups to execute the steps their persona might take to achieve their personal help-seeking objectives. In the workshops, all groups, irrespective of the persona assigned, began with an Internet search - typically via Google. However, the content of the search was variable, some groups searched specifically for mental health conditions such as depression or anxiety. Others inserted symptomrelated queries such as "heavy feeling in my body." Some groups reported being unsure of what to search for:
But she probably doesn't have a full understanding of what she has, I think like when she's searching, she's guessing.

Also, all participants were aware of limitations associated with search results, "... if there's nothing on the first page, you're screwed."

A common frustration in groups was to feel overwhelmed by the amount of results that a search returned ("Because a lot of people would feel that there's too much"). It is also worth noting that many final designs viewed an online search as a first step in seeking offline solutions (such as accessing traditional formal services).

\section{Personalisation of resources and autonomy}

There was a strong preference for online resources to provide both content and features that were personalised to young people. When groups browsed articles and information pages to learn more about the difficulty their persona was experiencing, they frequently indicated the need for tailored information and tools to suit the preference of the persona.

Not enough information. It just didn't do it for me. Not enough, not personal enough.

Personalisation was often achieved through a questionnaire or psychometric to help the young person address their current concerns. Participant's designs often included 'quizzes' to redirect users to personalised features. Importantly, personalisation included activities specific to their local area, books and documentaries relevant to their needs, and personal testimonies applicable to their challenges. The use of apps, diaries and mood-tracking technologies were also seen as part of an online resource that could be personally tailored to suit differing help-seeking needs of young people.

Supporting the young person's autonomy and avoidance of medicalized labels were also identified as important in supporting personalization:

The quiz doesn't diagnose you with depression or anxiety or anything, obviously, but it just sees where you're at. Then it would match you up a counsellor based on your results.

As noted above, relatedness was an important theme across all of the designs irrespective of the persona used. The importance of autonomy was again evident in this context. Anonymity was included across different design features, and was seen as important in personalized resources:

Think the anonymous aspect of it, where you don't need to give all of your information to get a diagnosis, where you can keep the app, but not have to give your name, your age, your location or anything like that unless you want to give your location. I think that makes people feel safer, without having to give personal details. 
Immediacy

In addition to chat, information, and personable features of online resources, participants also emphasized the need for help that could be acted upon immediately. In some instances, this took the form of practical activities that could be completed in times of stress, including guided meditation and breathing. Other features included daily challenges, inspirational quotes, and tips and tricks for self-help. One participant stated:

Maybe in the app, just having suggestions of things someone could do, just tips and tricks if they're feeling anxious or something. I don't know, something they could do at that moment, just to make them feel calmer.

It appears that some of the resources outlined so far - such as static information pages - fail to provide an additional step for online help-seekers, in that few offered suggestions for actions that could be taken in the moment to relieve immediate distress. Continuously, participants also mentioned games, guided relaxation, music, podcasts, and VR-led features as sources of instant activities.

\section{APPLYING THEORY TO UNDERSTAND ONLINE HELP. SEEKER'S NEEDS}

The studies outlined in this paper aimed to provide insight into how young people can be supported in their online helpseeking through the appropriate design of online mental health resources. Here we reflect on our findings via existing help-seeking and psychological theory, to highlight challenges and opportunities for design. Specifically, we discuss how the features and characteristics of online mental health resources can either facilitate or impede help-seeking. We first consider Rickwood's model and how it can be applied to understand the online help-seeking process.

\section{Rickwood's help-seeking model}

Online help-seeking appears to circumvent the necessity for the process to move from the intrapersonal to the interpersonal. Young people can engage in the entire helpseeking process, and successfully find help, without having to interact with another person should they choose not to. The Internet seems to offer options to support young people's preference for self-reliance.

In mapping online help-seeking to Rickwood's model, we can assume that the young person is exercising the expression stage by completing an online search. Search results inform the availability stage, whilst assessing and pursuing certain resources speaks to the willingness stage. This process can happen again once the young person has accessed an online resource, but perhaps in a different order. As the young person accesses different available resources, they are expressing their symptoms and identifying available sources of help. But they may also improve their mental health literacy through these actions, which in turn may facilitate increased awareness of their symptoms and difficulties. As young people build their mental health literacy, they also have on-going and progressive options on the degree to which they disclose their difficulty (i.e.

\begin{tabular}{|c|c|c|}
\hline & Facilitator & Barrier \\
\hline Awareness & $\begin{array}{l}\text { Personal stories, } \\
\text { articles and FAQ's }\end{array}$ & $\begin{array}{l}\text { Difficulty in accessing } \\
\text { relevant personalised content }\end{array}$ \\
\hline Expression & $\begin{array}{l}\text { Search, psychometric } \\
\text { assessments, trackers, } \\
\text { and questionnaires. }\end{array}$ & $\begin{array}{l}\text { Lack of mental health } \\
\text { literacy in order to input } \\
\text { appropriate search terms }\end{array}$ \\
\hline Availability & $\begin{array}{l}\text { Ease of access and } \\
\text { instantaneity }\end{array}$ & $\begin{array}{l}\text { Overwhelming number of } \\
\text { options of variable quality } \\
\text { available and difficulty in } \\
\text { choosing an appropriate } \\
\text { option }\end{array}$ \\
\hline Willingness & Anonymity & $\begin{array}{l}\text { Lack of live, immediate } \\
\text { support }\end{array}$ \\
\hline
\end{tabular}

Table 1. Applying Rickwood's model to design strategies that can either facilitate or impede the help-seeking process.

willingness). This might involve a transition from informal to formal sources of help. But equally it may mean remaining anonymous.

There are a number of features and characteristics that can be included in online resources to either facilitate or hinder online help-seeking in terms of this model. Table 1 outlines concrete examples. For example, while online search can facilitate expression, a lack of mental health literacy may act as a barrier to young people's ability to identify appropriate search terms. Structured questionnaires may help to overcome this barrier. Online resources that are designed to maximise the facilitators identified in Table 1 and overcome the listed barriers, have the potential to provide young people with a more satisfactory online help-seeking experience.

\section{Self-Determination Theory}

We have also proposed Self-Determination Theory as a useful framework to apply to conceptualise online helpseeking and meet the design requirements of young people. Many of the features and characteristics identified in this study can be thought of as meeting one of the three basic psychological needs. However, it is more likely that they will have an impact across multiple needs. Frustrating these needs can hinder help-seeking; much like satisfying these psychological needs can facilitate the process. In Table 2 we again outline concrete examples of how these needs can be met or, alternatively, frustrated in the online context.

Systems will work best when the three psychological needs are met, work together in harmony and facilitate one another. If not, the experience is more likely to be frustrating and not meet the help-seeker's needs. Our synthesised data consistently highlighted the need to effectively balance young people's needs for both competence and autonomy. To go online for help in the first instance can be theoretically understood as an autonomous act, leading to the exploration of resources that can build competencies, often in tandem with relatedness. However, while information pages can facilitate competency, information overload can reduce a young person's sense of competence. Participants indicated that information pages should have different sections, including facts and information that can be quickly 


\begin{tabular}{|c|c|c|}
\hline & Facilitator & Barrier \\
\hline Autonomy & Anonymity & Lack of personalised content \\
\hline Competency & $\begin{array}{l}\text { Accessible } \\
\text { information that can } \\
\text { promote different } \\
\text { activities. }\end{array}$ & $\begin{array}{l}\text { Lack of suggestions for } \\
\text { actions that can be completed } \\
\text { in the moment to relieve } \\
\text { distress }\end{array}$ \\
\hline Relatedness & $\begin{array}{l}\text { Live chat and } \\
\text { personal stories }\end{array}$ & $\begin{array}{l}\text { Unavailability of live formal } \\
\text { support } 24 / 7\end{array}$ \\
\hline
\end{tabular}

Table 2. Applying Self Determination Theory to design strategies that can either facilitate or impede basic psychological needs.

consumed; longer, more detailed sections; video; stories and images. Online help-seeking also meets the need for relatedness through a variety of different means (i.e. personal stories, discussion boards, chat). Providing choice within information pages and relatedness channels can help to support autonomy.

When considering the dominant theories of help-seeking, what is novel about online help-seeking in this study is that numerous needs and stages become realised through different individual features, sometimes simultaneously. For example, the preference for live anonymous chat with a professional can be seen as autonomously engaging in a competencybuilding activity with a human who can offer help. According to the presented theories, this would represent a propensity for both informal and formal sources, expression (Rickwood's help-seeking model) and a need for relatedness (SDT). However, the need for anonymity and the preference for this activity to only occur online directly challenges traditional ideas of relatedness. It raises the question: to what extent are young people seeking relatedness when favouring anonymised interventions online? Systems that use Rickwood's help-seeking model or Self-Determination Theory to guide their design should consider tensions such as those highlighted in this section.

\section{DESIGN RECOMMENDATIONS}

Consistent findings from both studies imply that online resources need to fulfil two practical roles, providing both referral tools and help sources. Irrespective of the helpseeker, and with a view to fulfilling these two needs, we propose four key recommendations: provide opportunities for connectedness; provide credible and accessible information; provide personalisation, but also respect anonymity; and, finally provide "just-in-time" support options. These recommendations are both theoretically informed and based on our data.

\section{Provide Opportunities for Connectedness}

Young people require online resources that offer opportunities to connect with others, peers and professionals, and feel part of a community. This can be achieved through various means including chat, forums and personal stories. Dependant on the stage of help-seeking the young person is at, connectedness will entail different types of activities. Previous work has shown the importance of providing structured, safe peer support channels for those seeking mental health support and the benefit this can have on young people's overall wellbeing $[39,46]$. There is value in providing opportunities for young people to discuss and ask questions from peers and professionals anonymously. Mental health concerns remain a very stigmatised topic and the preference for anonymous help-seeking online is understandable. This is also more likely to help young people move forward through the stages of help-seeking. Previous literature has confirmed that opportunities to find support online are now as important as traditional sources of support, both formal and informal [47]. The opportunity to feel part of a community online or engage with others in similar situations is an important need for young people when seeking help online. If this is designed correctly, helpseeker's need for relatedness and competence are met concurrently. Simultaneously, it also needs to be recognized that, for some help-seekers, there will be preferences to experience that connectedness in an offline context, but they will need assistance in finding appropriate offline opportunities. It is recommended that online resources include tools and mediums through which young people can experience connectedness in meaningful ways, including links to appropriate and, critically, local offline supports.

\section{Provide Credible and Accessible Information}

Findings from both studies have shown that online resources have to offer more than text-based mental health articles. Information needs to be offered in the form of different mediums including video, audio, short as well as long articles, and quicker to access FAQ sections. In this way, content can cater for various levels of mental health literacy and different online help-seeking goals. These findings reflect similar results to a study by Cunningham et al [13], which found that young people had different mental health information strategies. Mental health literacy has been found to be a significant barrier to help-seeking $[14,22,59]$ and technology offers opportunities to address this by providing content that suits different information preferences. In meeting varying informational preferences, online resources can also facilitate young people through the stages of helpseeking, whilst helping to meet their needs for competence and autonomy.

\section{Provide Personalisation, but also Respect Anonymity}

Young people are currently frustrated by the difficulty in accessing tailored and appropriate content relative to their needs. They feel overwhelmed by the abundance of choice and information that search results offer that do not speak to their online help-seeking goals. Their need for autonomy and competence are being frustrated. Other research has also shown that personalisation is an important factor for consideration when designing digital mental health technologies [25]. However, personalisation needs to take into account that anonymity is an important facilitator to help-seeking. This resonates with findings that in the case of search, as opposed to use of social media, users do not face the same temptations to self-censor [60]. Young people face a great deal of stigma regarding their mental health and when 
anonymity is included in the design of an online resource, users are likely to feel safer and more autonomous in disclosing their difficulties.

Finally, personalisation needs to recognize that different help-seekers will have different preferences for further support; how the need for relatedness is met will look different for different young people. Some help-seekers will prefer to have their entire help-seeking experience online, whilst others will go online with offline intent - for example, using a website to self-diagnose so as to make an online appointment with an appropriate local professional. Online resources that provide credible, tailored content are more likely to provide a positive help-seeking experience and designers of online mental health resources should endeavour to create personalised pathways for their target users.

\section{Provide "just-in-time" Support Options}

Online mental health resources have an important role to play in facilitating young people's help-seeking and ensuring that they access the appropriate help at the right time. In order to do this successfully, online resources need to have options that provide both immediate and long-term solutions. A study by de Alva and colleagues [2] investigating user's opinions of mental health apps found that users were often left feeling unsupported by mental health apps in times of distress. Similarly, O'Leary et al [45] found people who experience mental illnesses are often looking for "just-in-time" support when experiencing crisis. Participants in the co-design workshops indicated that providing online services and tools that are available and that are executable $24 / 7$ and that can relieve distress in the moment. The co-design workshops indicated that these might include chat with a professional in times of crisis or grounding techniques, but further investigation is needed into what types of exercises, activities and services would effectively meet this need.

\section{LIMITATIONS AND FUTURE WORK}

The personas in this paper represent three different helpseeking scenarios but should not be considered an exhaustive list - future research is likely to provide further detail and may identify other important help-seeking scenarios relevant to the online space. Additionally, while the scenarios and personas are applicable to differing help-seeking needs of a young person at various timepoints, it is not yet known how and why a young person might shift between help-seeking pathways. For example, a young person might only seek information online to self-educate; but they may also shift into preferring person-centred resources that provide relatedness or experience a crisis situation. The exact reasons for shifting between these scenarios and preferences warrants further investigation.

A noted in the related work section, this paper approached the issue of online help-seeking for mental health via the lens of help-seeking theory. In elaborating and refining our understanding of help seekers and scenarios, it will also be interesting to apply an Information Science perspective [17, $18,28]$. This was beyond the scope of the current paper but would provide alternative and complementary theories on how people seek, manage and use information. Longer-term research to synthesise theories of help-seeking and Information Science is likely to provide valuable insights on how young people use online resources to address mental health concerns. For example, application of Rieh's work on the judgement of information quality [53] could provide greater understanding of how young people assign credibility to online mental health resources.

Finally, our findings should be considered in light of the fact that, to the best of our knowledge, the sample was nonclinical. Therefore, it is not known if the results are applicable to clinical populations. As the study took place in Ireland our data is most representative of a western European demographic. Whilst the co-design study contained a near gender balance, the survey was disproportionately female. Future work should investigate if there are any gender differences regarding the help-seeking process of young people online.

\section{CONCLUSIONS}

Evidence suggests young people are increasingly using the Internet to access mental health information. The Internet allows them to access advice, help and support when they are experiencing distress and provides them with a wide variety of resources to choose from. The survey indicated that young people's goals varied when engaging in online help-seeking and the co-design study made use of personas to investigate these goals in terms of design needs. Both studies indicated that young people have concerns when looking for help online and that these concerns could be addressed through considered and purposeful design. Our research proposes that online mental health resources can facilitate the help-seeking process by providing (1) opportunities for connectedness (2) credible and accessible information (3) personalisation, but also respect autonomy and (4) "just-in-time" support options. The contributions of this work are an understanding of how young people use the Internet to engage in online helpseeking behaviour and the recognition of specific design opportunities to positively facilitate online help-seeking. Offline help-seeking faces a number of barriers, including stigma, the opportunity now exists to address these in an online context.

\section{ACKNOWLEDGMENTS}

This project is part of TEAM (Technology Enabled Mental Health for Young People). TEAM has received funding from the European Union's Horizon 2020 research and innovation program under the Marie Skłodowska-Curie grant agreement No. 722561.

\section{REFERENCES}

[1] Icek Ajzen. 1985. From Intentions to Actions: A Theory of Planned Behavior. In Action Control. Springer Berlin Heidelberg, 11-39.

[2] Fernando Estrada Martinez de Alva, Greg Wadley, and Reeva Lederman. 2015. It feels different from real life. Proceedings of the Annual Meeting of the Australian 
Special Interest Group for Computer Human Interaction on - OzCHI '15, ACM Press, 598-602. DOI :

https://doi.org/10.1145/2838739.2838806

[3] Nazanin Andalibi, Pinar Ozturk, and Andrea Forte. 2015. Depression-related Imagery on Instagram. Proceedings of the 18th ACM Conference Companion on Computer Supported Cooperative Work \& Social Computing - CSCW'15 Companion, ACM Press, 231234. DOI : https://doi.org/10.1145/2685553.2699014

[4] Paul Best, Elena Gil-Rodriguez, Roger Manktelow, and Brian J Taylor. 2016. Seeking help from everyone and no-one: Conceptualizing the online help-seeking process among adolescent males. Qualitative Health Research 26, 8: 1067-1077. DOI: https://doi.org/10.1177/1049732316648128

[5] Michael L. Birnbaum, Asra F. Rizvi, Christoph U. Correll, John M. Kane, and Jamie Confino. 2017. Role of social media and the Internet in pathways to care for adolescents and young adults with psychotic disorders and non-psychotic mood disorders. Early Intervention in Psychiatry 11, 4: 290-295. DOI:

https://doi.org/10.1111/eip.12237

[6] Victoria Blake, Kerrie Buhagiar, Sylvia Kauer, Mariesa Nicholas, Lena Sanci, and Julie Grey. 2016. Using participatory design to engage young people in the development of a new online tool to increase helpseeking. Journal of Applied Youth Studies 1, 3: 68-83.

[7] Lisa M. Bohon, Kelly A. Cotter, Richard L. Kravitz, Philip C. Cello, and Erik Fernandez y Garcia. 2016. The Theory of Planned Behavior as it predicts potential intention to seek mental health services for depression among college students. Journal of American College Health 64, 8: 593-603. DOI: https://doi.org/10.1080/07448481.2016.1207646

[8] Virginia Braun and Victoria Clarke. 2006. Using thematic analysis in psychology. Qualitative Research in Psychology 3, 2: 77-101. DOI: 10.1191/1478088706qp063oa

[9] Jane M. Burns, Emma Birrell, Marie Bismark, et al. 2016. The role of technology in Australian youth mental health reform. Australian Health Review 40, 5: 584. DOI: 10.1071/AH15115

[10] Rafael A. Calvo and Dorian Peters. 2014. Positive computing : technology for wellbeing and human potential. The MIT Press.

[11] Munmun De Choudhury, Scott Counts, and Eric Horvitz. 2013. Social Media as a Measurement Tool of Depression in Populations. In Proceedings of the 5th Annual ACM Web Science Conference (WebSci '13). ACM Press, 47-56. DOI: https://doi.org/10.1145/2567948.2580064

[12] Stephan Collishaw. 2015. Annual research review: Secular trends in child and adolescent mental health. Journal of Child Psychology and Psychiatry and Allied
Disciplines 56, 3: 370-393.

[13] Charles E. Cunningham, John R. Walker, John D. Eastwood, et al. 2014. Modeling mental health information preferences during the early adult years: A discrete choice conjoint experiment. Journal of Health Communication 19, 4: 413-440. DOI: 10.1080/10810730.2013.811324

[14] Kate Daine, Keith Hawton, Vinod Singaravelu, Anne Stewart, Sue Simkin, and Paul Montgomery. 2013. The power of the web: a systematic review of studies of the influence of the internet on self-harm and suicide in young people. PloS one 8, 10: 1-6. DOI: https://doi.org/10.1371/journal.pone.0077555

[15] Rikke Dam and Teo Siang. Empathy Map - Why and How to Use It | Interaction Design Foundation. Retrieved 15 September 2019 from https://www.interactiondesign.org/literature/article/empathy-map-why-andhow-to-use-it.

[16] Edward L Deci and Richard M Ryan. 2009. The What and Why of Goal Pursuits: Human Needs and the SelfDetermination of Behavior. Psychological Inquiry, 11(4), 227-268. DOI: https://doi.org/10.1207/S15327965PLI1104_01

[17] Karen E. Fisher, Sandra Erdelez, and Lynne McKechnie. 2005. Theories of Information Behavior. Information Today, Medford, NJ.

[18] Karen E. Fisher and Heidi Julien. 2009. Information behavior. Annual Review of Information Science and Technology 43, 1: 1-73. DOI: https://doi.org/10.1002/aris.2009.1440430114

[19] Mareka Frost and Leanne Casey. 2016. Who Seeks Help Online for Self-Injury? Archives of Suicide Research 20, 1: 69-79. DOI: https://doi.org/10.1080/13811118.2015.1004470

[20] L Kris Gowen. 2013. Online mental health information seeking in young adults with mental health challenges. Journal of Technology in Human Services 31, 2: $97-$ 111. DOI: https://doi.org/10.1080/15228835.2013.765533

[21] Amelia Gulliver, Kathleen M. Griffiths, Helen Christensen, et al. 2012. Internet-based interventions to promote mental health help-seeking in elite athletes: An exploratory randomized controlled trial. Journal of Medical Internet Research 14, 3: 120-137. DOI: 10.2196/jmir.1864

[22] Amelia Gulliver, Kathleen M Griffiths, and Helen Christensen. 2012. Barriers and facilitators to mental health help-seeking for young elite athletes: A qualitative study. BMC Psychiatry 12: 1. DOI: https://doi.org/10.1186/1471-244X-12-157

[23] Penny Hagen, Philippa Collin, Atari Metcalf, Mariesa Nicholas, and Kitty Rahilly. 2012. Participatory Design of evidence-based online youth mental health 
promotion, intervention and treatment. A Young and

Well Cooperative Research Centre innovative

methodologies guide. Retrieved from

http://pandora.nla.gov.au/pan/141862/20160405-

1343/www.youngandwellcrc.org.au/wp-

content/uploads/2014/03/Young_and_Well_CRC_IM_P

D_Guide.pdf

[24] Sarah Elisabeth Hetrick, Jo Robinson, Eloise Burge, et al. 2018. Youth Codesign of a Mobile Phone App to Facilitate Self-Monitoring and Management of Mood Symptoms in Young People With Major Depression, Suicidal Ideation, and Self-Harm. JMIR Mental Health 5, 1: e9. DOI: 10.2196/mental.9041

[25] Chris Hollis, Richard Morriss, Jennifer Martin, et al. 2015. Technological innovations in mental healthcare: harnessing the digital revolution. British Journal of Psychiatry 206, 4: 263-265. DOI:

10.1192/bjp.bp.113.142612

[26] Tharon Howard and Tharon. 2014. Journey mapping. Communication Design Quarterly Review 2, 3: 10-13. DOI: https://doi.org/10.1145/2644448.2644451

[27] Hsiu-Fang Hsieh and Sarah E. Shannon. 2005. Three Approaches to Qualitative Content Analysis. Qualitative Health Research 15, 9: 1277-1288. DOI: https://doi.org/10.1177/1049732305276687

[28] Bernard J. Jansen and Soo Young Rieh. 2010. The seventeen theoretical constructs of information searching and information retrieval. Journal of the American Society for Information Science and Technology 61, 8: 1517-1534. DOI: https://doi.org/10.1002/asi.21358

[29] Gillian Karwig, Derek Chambers, and Fenella Murphy. 2015. Reaching Out in College: Help-Seeking at Third Level in Ireland, ReachOut Ireland. Dublin.

[30] Sylvia Kauer, Kerrie Buhagiar, and Lena Sanci. 2017. Facilitating mental health help seeking in young adults: The underlying theory and development of an online navigation tool. Advances in Mental Health 15, 1: 7187. DOI: $10.1136 /$ bmjopen-2016-015303

[31] Sylvia Deidre Kauer, Cheryl Mangan, and Lena Sanci. 2014. Do online mental health services improve helpseeking for young people? A systematic review. Journal of Medical Internet Research 16, 3: 314-331. DOI: 10.2196/jmir.3103

[32] Maria Kauhondamwa, Heike Winschiers-Theophilus, Simson Kapembe, et al. 2018. Co-creating personal augmented reality accessories to enhance social wellbeing of urban San youth. ACM International Conference Proceeding Series, Association for Computing Machinery, 99-108. DOI: https://doi.org/10.1145/3283458.3283480

[33] Ronald C Kessler, G Paul Amminger, Sergio AguilarGaxiola, Jordi Alonso, Sing Lee, and T Bedirhan Ustun. 2007. Age of onset of mental disorders: A review of recent literature. Current Opinion in Psychiatry 20, 4: 359-364. DOI: 10.1097/YCO.0b013e32816ebc8c

[34] Becky Mars, Jon Heron, Lucy Biddle, et al. 2015. Exposure to, and searching for, information about suicide and self-harm on the Internet: Prevalence and predictors in a population based cohort of young adults. Journal of Affective Disorders 185: 239-245. DOI: 10.1016/j.jad.2015.06.001

[35] Nicola Marsden, Monika Pröbster, Mirza Ehsanul Haque, and Julia Hermann. 2017. Cognitive styles and personas. Proceedings of the 29th Australian Conference on Computer-Human Interaction - OZCHI '17, ACM Press, 452-456. DOI: https://doi.org/10.1145/3152771.3156156

[36] Tara Matthews, Tejinder Judge, and Steve Whittaker. 2012. How do designers and user experience professionals actually perceive and use personas? Proceedings of the 2012 ACM annual conference on Human Factors in Computing Systems - CHI '12, ACM Press, 1219. DOI: https://doi.org/10.1145/2207676.2208573

[37] Jörn Moock. 2014. Support from the Internet for Individuals with Mental Disorders: Advantages and Disadvantages of e-Mental Health Service Delivery. Frontiers in Public Health 2, 65. DOI: https://doi.org/10.3389/fpubh.2014.00065

[38] Swenda Moreh and Henry O'Lawrence. 2016. Common Risk Factors Associated With. Journal of Health \& Human Services Administration 39, 2: 283-310.

[39] Khushnood Naqshbandi, David N. Milne, Ben Davies, Sophie Potter, Rafael A. Calvo, and Simon Hoermann. 2016. Helping young people going through tough times. Proceedings of the 28th Australian Conference on Computer-Human Interaction - $\mathrm{OzCHI}$ '16, ACM Press, 640-642. DOI: https://doi.org/10.1145/3010915.3011848

[40] Mariesa Nicholas, Penny Hagen, Kitty Rahilly, and Nathalie Swainston. 2012. Using participatory design methods to engage the uninterested. Proceedings of the 12th Participatory Design Conference on Exploratory Papers Workshop Descriptions Industry Cases - Volume 2 - PDC '12, ACM Press, 121. DOI: https://doi.org/10.1145/2348144.2348183

[41] Lene Nielsen. 2004. Engaging Personas and Narrative Scenarios. PhD series, Samfundslitteratur, 17.

[42] Lene Nielsen. 2010. Personas in Co-creation and Codesign. Proceedings of the 11th Danish HumanComputer Interaction Research Symposium (DHRS2011). Copenhagen Business School Press, 3840.

[43] Lene Nielsen. 2013. Personas |. The Encyclopedia of Human-Computer Interaction, 2nd Ed. Retrieved 15 December 2018 from https://www.interactiondesign.org/literature/book/the-encyclopedia-of-human- 
computer-interaction-2nd-

ed/personas\#heading_Four_different_perspectives_page _12414.

[44] Lene Nielsen. 2013. Personas - User Focused Design. Springer London, London.

[45] Kathleen O'Leary, Arpita Bhattacharya, Sean A. Munson, Jacob O. Wobbrock, and Wanda Pratt. 2017. Design Opportunities for Mental Health Peer Support Technologies. Proceedings of the 2017 ACM Conference on Computer Supported Cooperative Work and Social Computing - CSCW '17, ACM Press, 14701484. DOI: https://doi.org/10.1145/2998181.2998349

[46] Kathleen O'Leary, Stephen M Schueller, Jacob O Wobbrock, and Wanda Pratt. 2018. 'Suddenly, we got to become therapists for each other': Designing Peer Support Chats for Mental Health. In Proceedings of the 2018 CHI Conference on Human Factors in Computing Systems (CHI '18). Association for Computing Machinery, New York, NY, USA, Paper 331, 1-14. DOI: https://doi.org/10.1145/3173574.3173905

[47] Sun Young Park and Sun Young. 2018. Social Support Mosaic. Proceedings of the 2018 on Designing Interactive Systems Conference 2018 - DIS '18, ACM Press, 121-133. DOI: https://doi.org/10.1145/3196709.3196787

[48] Dorian Peters, Rafael A. Calvo, and Richard M. Ryan. 2018. Designing for Motivation, Engagement and Wellbeing in Digital Experience. Frontiers in Psychology 9: 797. DOI: https://doi.org/10.3389/fpsyg.2018.00797

[49] Claudette Pretorius, Derek Chambers, Benjamin Cowan, and David Coyle. 2019. Young People Seeking Help Online for Mental Health: Cross-Sectional Survey Study. JMIR mental health 6, 8: e13524. DOI: $10.2196 / 13524$

[50] Claudette Pretorius, Derek Chambers, and David Coyle. 2019. Young People's Online Help-Seeking and Mental Health Difficulties: Systematic Narrative Review. Journal of medical Internet research 21, 11: e13873. DOI: $10.2196 / 13873$

[51] Debra Rickwood, Frank P Deane, Coralie J Wilson, and Joseph Ciarrochi. 2005. Young people's help-seeking for mental health problems. Australian e-Journal for the Advancement of Mental Health 4, 3: 218-251. DOI: https://doi.org/10.5172/jamh.4.3.218
[52] Debra Rickwood, Kerry Thomas, and Sally Bradford. 2012. Help-seeking measures in mental health: a rapid review. The Sax Institute, Australia

[53] Soo Young Rieh. 2002. Judgment of information quality and cognitive authority in the Web. Journal of the American Society for Information Science and Technology 53, 2: 145-161. DOI: https://doi.org/10.1002/asi.10017

[54] Richard M Ryan and Edward L Deci. 2005. SelfDetermination Theory and the Facilitation of Intrinsic Motivation, Social Development, and Well-Being SelfDetermination Theory. The American psychologist, 55, 68-78. DOI: 10.1037//0003-066x.55.1.68

[55] Keziban Salaheddin and Barbara Mason. 2016. Identifying barriers to mental health help-seeking among young adults in the UK:a cross-sectional survey. British Journal of General Practice 66, 651: e686-e692. DOI: https://doi.org/10.3399/bjgp16X687313

[56] Will Simm, Maria Angela Ferrario, Adrian Gradinar, et al. Anxiety and Autism: Towards Personalized Digital Health. In Proceedings of the 2016 CHI Conference on Human Factors in Computing Systems (CHI '16). ACM. Press, 1270-1281. DOI: https://doi.org/10.1145/2858036.2858259

[57] Beth St Jean, Mega Subramaniam, Natalie Greene Taylor, Rebecca Follman, Christie Kodama, and Dana Casciotti. 2015. The influence of positive hypothesis testing on youths' online health-related information seeking. New Library World Journal of Documentation Iss Journal of Documentation 116, 3: 136-154.

[58] Harvey A Whiteford, Louisa Degenhardt, Jürgen Rehm, et al. 2013. Global burden of disease attributable to mental and substance use disorders: findings from the Global Burden of Disease Study 2010. The Lancet 382, 9904: 1575-1586. DOI: $10.1016 / \mathrm{S} 0140-$ 6736(13)61611-6

[59] Coralie J Wilson, Frank P Deane, Joseph V Ciarrochi, and Debra Rickwood. 2005. Measuring help seeking intentions: Properties of the General Help Seeking Questionnaire. Canadian Journal of Counselling 39, 1: 15-28. DOI: https://doi.org/10.5172/jamh.4.3.218

[60] Anis Zaman, Rupam Acharyya, Henry Kautz, and Vincent Silenzio. 2019. Detecting Low Self-Esteem in Youths from Web Search Data. In The World Wide Web Conference ( $W W W$ '19). ACM Press, 2270-2280. DOI: https://doi.org/10.1145/3308558.3313557 